PHYSICAL REVIEW B 71, 169904(E) (2005)

\title{
Erratum: Dynamical exchange-correlation potentials for an electron liquid [Phys. Rev. B 65, 235121 (2002)]
}

\author{
Zhixin Qian and Giovanni Vignale \\ (Received 16 February 2005; published 26 April 2005)
}

DOI: 10.1103/PhysRevB.71.169904

PACS number(s): 71.15.Mb, 71.10.-w, 71.45.Gm, 99.10.Cd

The value of $\mu_{x c}=0.00851$ at $r_{s}=5$ in Table $\mathrm{I}$ is erroneous. The correct value is $\mu_{x c}=0.00869$.

We acknowledge M. van Faassen for pointing out the error. 